\title{
Tracking the origin of Earth's volatile elements depletion with indium
}

\author{
DEZE LIU ${ }^{1}$, FRÉDÉRIC MOYNIER ${ }^{2}$, JULIEN SIEBERT ${ }^{3}$ \\ AND PAOLO A. SOSSI ${ }^{4}$ \\ ${ }^{1}$ Université de Paris, Institut de Physique du Globe de Paris, \\ CNRS UMR7154 \\ ${ }^{2}$ Université de Paris, Institut de Physique du Globe de Paris, \\ CNRS \\ ${ }^{3}$ Institut de Physique du Globe de Paris \\ ${ }^{4}$ ETH Zürich \\ Presenting Author: liudeze@ipgp.fr
}

The abundances of volatile elements provide insight into fundamental processes of planetary accretion and differentiation. In chondrites, the abundances of volatile elements vary primarily as a function of the temperature at which $50 \%$ of their mass has calculated to condense during cooling of the solar nebula $\left(\mathrm{T}_{\mathrm{C}}{ }^{50}\right)$. In contrast, core formation also plays an important role in controlling the volatile element depletion in the bulk silicate Earth (BSE). Indium is a siderophile and volatile element. Its terrestrial abundance paradoxically lies above the abundance vs. $\mathrm{T}_{\mathrm{C}}{ }^{50}$ trend defined by lithophile volatile elements in the BSE [1]. Notably, the use of $\mathrm{T}_{\mathrm{C}}{ }^{50}$ presumes depletion took place under nebular conditions [2], and hence may not be an appropriate description of the volatility of indium under more oxidizing- and higher pressure conditions during chondrule formation and postnebular processes.

To constrain the volatility of In from silicate melts under conditions close to chondrule and planetary formation, we performed evaporation experiments on an In-doped basaltic melt under variable oxygen fugacities $\left(f \mathrm{O}_{2}\right)$ and temperatures $(T)$. Elemental volatility is quantified by deriving the enthalpy and entropy during evaporation at the given conditions $\left(T, f \mathrm{O}_{2}\right.$ and duration)[3].

Our results show that the volatility of indium strongly increases with decreasing $f \mathrm{O}_{2}$, as observed for other moderately volatile elements $[3,4]$ and is relatively less volatile than $\mathrm{Zn}$ for $\mathrm{fO}_{2}$ typical of planetary mantles. In spite of this new volatility scale, In is no more depleted than $\mathrm{Zn}$ in the BSE notwithstanding its more siderophile nature. Therefore, the chondritic $\mathrm{Zn} / \mathrm{In}$ ratio in the BSE is either $i$ ) a coincidence or ii) consistent with a 'hockey stick' volatile depletion pattern [5]. The lower volatility of In relative to $\mathrm{Zn}$ supports the idea that its over-abundance in the BSE may be inherited from its source materials, which have a CI-like ratio for volatile elements whose $\mathrm{T}_{\mathrm{C}}{ }^{50}<750 \mathrm{~K}$ [6].

[1] Witt-Eickschen et al. (2009) GCA 73, 1755-1778

[2] Lodders. (2003) Astrophys.J. 591, 1220-1247

[3] Sossi et al. (2019) GCA 260, 204-231

[4] Norris and Wood. (2017) Nature 549, 507

[5] Braukmüller et al. (2019) Nat. Geosci. 12, 564-568

[6] Hellmann et al. (2020) EPSL 549, 116508 